INSTITUT NATIONAL DE RECHERCHE EN INFORMATIQUE ET EN AUTOMATIQUE

\title{
On parabolic and elliptic spectral dichotomy
}

A.N. Malyshev , M. Sadkane

$\mathbf{N}^{\circ} 2332$

juillet 1994

PROGRAMME 6

\section{apport}

de recherche 



\title{
RINRIA
}

\section{On parabolic and elliptic spectral dichotomy}

\author{
A.N. Malyshev * , M. Sadkane ** \\ Programme 6 - Calcul scientifique, modélisation et logiciel numérique \\ Projet ALADIN
}

Rapport de recherche $\mathrm{n}^{\circ} 2332$ - juillet $1994-13$ pages

\begin{abstract}
We discuss two spectral dichotomy techniques: one for computing an invariant subspace of a nonsymmetric matrix associated with the eigenvalues inside and outside a given parabola. Another for computing a right deflating subspace of a regular matrix pencil associated with the eigenvalues inside and outside a given ellipse. The techniques use matrices of order twice the order of the original matrices on which the spectral dichotomy by the unit circle and by the imaginary axis apply efficiently. We prove the equivalence between the condition number of the original problems and that of the transformed ones.
\end{abstract}

Key-words: spectral dichotomy, spectral transformation, spectral condition number

\footnotetext{
*Institute of Mathematics, Novosibirsk, 630090, Russia (malyshev@math.nsk.su). This work was carried out while the author was visiting IRISA. The work was supported by the French Ministry of Defense (DRET) and partially by Russian Fund of Fundamental Researches (93-011-1515)

**sadkane@irisa.fr
} 


\section{Sur la dichotomie spectrale par une parabole et par une ellipse}

Résumé : Nous discutons deux techniques de dichotomie spectrale: une pour calculer un sous-espace invariant d'une matrice non symétrique, associé aux valeurs propres à l'intérieur et à l'extérieur d'une parabole. L'autre pour calculer un sous espace invariant droit d'un faisceau régulier de matrices, associé aux valeurs propres à l'intérieur et à l'extérieur d'une ellipse. Ces méthodes sont basées sur la construction de matrices d'ordre deux fois celui des matrices d'origine, et sur lesquelles les techniques de dichotomie spectrale par le cercle unité et par l'axe imaginaire s'appliquent efficacement. Nous montrons l'equivalence entre le conditionnement spectral du problème original et celui du problème transformé.

Mots-clé : dichotomie spectrale, transformation spectrale, conditionnement spectral 


\section{Introduction}

In this note we are concerned with some spectral transformations for computing invariant subspaces corresponding to the eigenvalues of a matrix (pencil) in a given domain $\mathcal{D}$ of the complex plane.

We consider first the case where $\mathcal{D}$ is the interior (exterior) of a given parabola $\gamma$ and where we seek an invariant subspace of a matrix $A$ having no eigenvalues on $\gamma$. By using a special matrix $\mathcal{A}$, we show that we reduce the problem to that of the computation of an invariant subspace of $\mathcal{A}$ corresponding to the left (right) half plane of the complex plane. Therefore the results concerning the spectral dichotomy by the imaginary axis $[4,6,7,8]$ can be applied efficiently.

In the second part we consider the case where $\mathcal{D}$ is the interior (exterior) of an ellipse $\Gamma$ and where we seek an invariant subspace of a regular matrix pencil $\lambda B-A$ having no eigenvalues on $\Gamma$. By using a special regular matrix pencil $\lambda \mathcal{B}-\mathcal{A}$, we show that this problem is reduced to that of the computation of an invariant subspace of the pencil $\lambda \mathcal{B}-\mathcal{A}$ corresponding to the interior (exterior) of the unit circle. Therefore the results concerning the circular spectral dichotomy $[2,6,7,8]$ can be applied efficiently. This latter problem has recently been studied in [5]. We propose here a simple solution based on standard linear algebra without making use of Green matrices.

We study the condition numbers of these two problems and prove the equivalence between them and those of the transformed problems.

Throughout this note, $\|x\|$ denotes the Euclidean norm of the vector $x$. $\|X\|$ and $X^{*}$ denote the spectral norm and the conjugate transpose of the matrix $X$. We denote by $I_{n}$ the identity matrix of order $n$.

\section{Parabolic dichotomy}

Let us consider a parabola $\gamma$ in the $(x, y)$ plane satisfying the equation

$$
y^{2}=2 p(p / 2-x)
$$

where $p>0$ is some real parameter. This parabola has its center at the origin and its branches go to the left half-plane symmetrically with respect to the real axis.

Consider the mapping $\varphi: \lambda \in \mathbb{C} \mapsto z \in \mathbb{C}$ defined by the formula $z=$ $(\lambda+\sqrt{p / 2})^{2}$. If we write $z=x+i y$ then we obtain

$$
x=(\Re \lambda+\sqrt{p / 2})^{2}-(\Im \lambda)^{2} \text { and } y=2(\Re \lambda+\sqrt{p / 2}) \Im \lambda .
$$

RR $\mathrm{n}^{\circ} 2332$ 
It follows that $y^{2}=4(\Re \lambda+\sqrt{p / 2})^{2}\left[(\Re \lambda+\sqrt{p / 2})^{2}-x\right]$. Thus $\varphi$ bijectively maps the straight line $\Re \lambda=c \in \mathbf{R}$ onto the parabola $y^{2}=2 \bar{p}(\bar{p} / 2-x)$ with $\bar{p}=$ $2(c+\sqrt{p / 2})^{2}$. This family of parabolae depending on the parameter $\bar{p}$ has the same center at the origin. In particular, $\varphi$ bijectively maps the imaginary axis onto the parabola $\gamma$. It is easy to see that $\varphi$ maps the region

$$
\{\lambda \in \mathbb{C} \mid \Re \lambda<-2 \sqrt{p / 2}\} \cup\{\lambda \in \mathbb{C} \mid \Re \lambda>0\}
$$

onto the exterior of $\gamma$ and maps the strip

$$
\{\lambda \in \mathbb{C} \mid-2 \sqrt{p / 2}<\Re \lambda<0\}
$$

onto the interior of $\gamma$. The restriction of the mapping $\varphi$ on the set

$$
\Omega=\{\lambda \in \mathbb{C} \mid \Re \lambda>-\sqrt{p / 2}\}
$$

is 1 -to- 1 and $\varphi(\Omega)=\{\lambda \in \mathbb{C} \mid(\Re \lambda>0) \operatorname{or}(\Im \lambda \neq 0)\}$, that is $\varphi(\Omega)$ is the whole $(x, y)$ plane with the real negative axis deleted. Finally it is easy to see that the right halfplane, i.e. the set of $\lambda$ with $\Re \lambda>0$ is mapped conformally onto the exterior of the parabola $y^{2}=2 p(p / 2-x)$.

After this preliminaries we consider now a matrix $A$ of order $n$ having no eigenvalue on the parabola $\gamma$ of equation $y^{2}=2 p(p / 2-x)$. Let $\mathcal{A}$ be the matrix of order $2 n$ defined by

$$
\mathcal{A}=\left[\begin{array}{cc}
-\sqrt{\frac{p}{2}} I_{n} & A \\
I_{n} & -\sqrt{\frac{p}{2}} I_{n}
\end{array}\right] .
$$

It is easy to see that the eigenvalues $\lambda$ of $\mathcal{A}$ and the eigenvalues $z$ of $A$ satisfy

$$
z=(\lambda+\sqrt{p / 2})^{2}
$$

$¿$ From the above discussion about the properties of the mapping $\varphi$ and the assumption about the eigenvalues of $A$, we see that the matrix $\mathcal{A}$ has no eigenvalue on the imaginary axis. Thus the problem of verification of absence of the eigenvalues of the matrix $A$ on the parabola $\gamma$ is transformed to the spectral dichotomy problem with respect to the imaginary axis. This latter problem has been deeply studied from theoretical and practical aspects $[4,7,8]$.

We assume from now on that $\|A\|=1$. This assumption is not restrictive since otherwise we can take $A_{1}=\frac{1}{\|A\|} A$ and $p_{1}=\frac{p}{\|A\|}$. 
In the spectrum dichotomy problem for the matrix $\mathcal{A}$ with respect to the imaginary axis, the quality of the dichotomy is characterized by the numerical parameter $[7,1]$

$$
\tilde{\alpha}=\sup _{\Re \lambda=0}\left\|\left(\lambda I_{2 n}-\mathcal{A}\right)^{-1}\right\| .
$$

This parameter has already been used in the context of matrix stability [9]. Similarly, the quality of the dichotomy for the matrix $A$ with respect to the ellipse $\gamma$ is characterized by the parameter

$$
\alpha=\sup _{z \in \gamma}\left\|\left(z I_{n}-A\right)^{-1}\right\|
$$

The natural question that one may ask is how relate the two quantities $\alpha$ and $\tilde{\alpha}$. The answer is given in the following proposition

Proposition 2.1 Let $\alpha$ and $\tilde{\alpha}$ be the two parameters defined in (5) and (6). Then

$$
\alpha \leq \tilde{\alpha} \leq \alpha+\sqrt{\alpha} \sqrt{1+\alpha}
$$

Proof : We have

$$
\begin{aligned}
\left(\lambda I_{2 n}-\mathcal{A}\right)^{-1}= & {\left[\begin{array}{cc}
\left(\lambda+\sqrt{\frac{p}{2}}\right) I_{n} & A \\
I_{n} & \left(\lambda+\sqrt{\frac{p}{2}}\right) I_{n}
\end{array}\right] } \\
& \times\left[\begin{array}{cc}
\left(\lambda+\sqrt{\frac{p}{2}}\right)^{2} I_{n}-A & 0 \\
0 & \left(\lambda+\sqrt{\frac{p}{2}}\right)^{2} I_{n}-A
\end{array}\right]^{-1} .
\end{aligned}
$$

By considering the $n \times n$ block in position $(2,1)$ of the resulting matrix, we obtain

$$
\tilde{\alpha}=\sup _{\Re \lambda=0}\left\|\left(\lambda I_{2 n}-\mathcal{A}\right)^{-1}\right\| \geq \sup _{z \in \gamma}\left\|\left(z I_{n}-A\right)^{-1}\right\|=\alpha .
$$

The use of (4) gives

$$
\left\|\left(\lambda I_{2 n}-\mathcal{A}\right)^{-1}\right\| \leq\left\|\left(\begin{array}{cc}
\sqrt{z} I_{n} & A \\
I_{n} & \sqrt{z} I_{n}
\end{array}\right)\right\|\left\|\left(z I_{n}-A\right)^{-1}\right\|,
$$

therefore, due to the fact that $\|A\|=1$ we have

$$
\left\|\left(\lambda I_{2 n}-\mathcal{A}\right)^{-1}\right\| \leq\left\|\left(z I_{n}-A\right)^{-1}\right\|(1+\sqrt{|z|}) .
$$

$\operatorname{RR} n^{\circ} 2332$ 
We deduce that if $|z| \leq \frac{1+\alpha}{\alpha}$, then $\left\|\left(\lambda I_{2 n}-\mathcal{A}\right)^{-1}\right\| \leq \alpha\left(\sqrt{\frac{1+\alpha}{\alpha}}+1\right)=\alpha+\sqrt{\alpha} \sqrt{1+\alpha}$. Now if $|z|>\frac{1+\alpha}{\alpha}$, the formula $\left(z I_{n}-A\right)^{-1}=\frac{1}{z} I_{n}+\frac{1}{z} A\left(z I_{n}-A\right)^{-1}$ yields

$$
\begin{aligned}
\left\|\left(\lambda I_{2 n}-\mathcal{A}\right)^{-1}\right\| & \leq\left(\left\|\left(z I_{n}-A\right)^{-1}\right\|+1\right) \frac{\sqrt{|z|}+1}{|z|} \\
& \leq(\alpha+1)\left(\frac{1}{|z|}+\frac{1}{\sqrt{|z|}}\right)<\alpha+\sqrt{\alpha} \sqrt{1+\alpha}
\end{aligned}
$$

Thus provided that $\alpha$ is not small, the quality of the spectral dichotomy of the matrix $A$ with respect to the parabola $\gamma$ and that of the matrix $\mathcal{A}$ with respect to the imaginary axis are equivalent.

We now describe how to obtain a solution to the spectral dichotomy problem for $A$ with respect to the parabola $\gamma$ having calculated a solution to the spectral dichotomy problem for $\mathcal{A}$ with respect to the imaginary axis. Let $P \in \mathbf{C}^{\mathbf{n} \times \mathbf{n}}$ be the projection matrix onto the right eigenspace of $A$ associated with the eigenvalues outside the parabola $\gamma$ and $\mathcal{P} \in \mathbf{C}^{\mathbf{2 n \times 2 n}}$ the projection matrix onto the right eigenspace of $\mathcal{A}$ associated with the eigenvalues on the right half-plane of the complex plane. The following proposition characterizes the relation between $P$ and $\mathcal{P}$.

Proposition 2.2 Let us partition $\mathcal{P}$ in the following form

$$
\mathcal{P}=\left(\begin{array}{cc}
\mathcal{P}_{1} & \mathcal{P}_{2} \\
\mathcal{P}_{3} & \mathcal{P}_{4}
\end{array}\right) \quad \text { with } \quad \mathcal{P}_{i} \in \mathbf{C}^{\mathbf{n} \times \mathbf{n}}, \quad i=1,4
$$

Then

$$
P=2 \mathcal{P}_{1}=2 \mathcal{P}_{4}=4 \mathcal{P}_{2} \mathcal{P}_{3}
$$

Moreover

$$
\mathcal{P}_{2}=\frac{1}{2}(P A)^{\frac{1}{2}} .
$$

Proof : Suppose that the matrix $A$ is nonsingular (below this restriction will be removed). Let us define a solution $X$ to the matrix equation

$$
\left(X+\sqrt{\frac{p}{2}} I_{n}\right)^{2}=A
$$

whose eigenvalues are assumed to be in the domain

$$
\mathcal{D}=\{\lambda \in \mathbb{C} \mid \Re \lambda>-\sqrt{p / 2}\} \cup\{\lambda \in \mathbb{C} \mid \Re \lambda=-\sqrt{p / 2} \& \Im \lambda>0\} .
$$


It is clear that such matrix $X$ exists and is uniquely defined ${ }^{1}$. Notice that the matrix $X_{1}=-X-2 \sqrt{p / 2} I_{n}$ also satisfies the equation $\left(X_{1}+\sqrt{p / 2} I_{n}\right)^{2}=A$.

Then the matrix $\mathcal{A}$ can be decomposed in the following form:

$$
\begin{aligned}
\mathcal{A}= & \left(\begin{array}{cc}
X+\sqrt{\frac{p}{2}} I_{n} & -X-\sqrt{\frac{p}{2}} I_{n} \\
I_{n} & I_{n}
\end{array}\right)\left(\begin{array}{cc}
X & 0 \\
0 & -X-2 \sqrt{\frac{p}{2}} I_{n}
\end{array}\right) \\
& \times\left(\begin{array}{cc}
\frac{1}{2}\left(X+\sqrt{\frac{p}{2}} I_{n}\right)^{-1} & \frac{1}{2} I_{n} \\
-\frac{1}{2}\left(X+\sqrt{\frac{p}{2}} I_{n}\right)^{-1} & \frac{1}{2} I_{n}
\end{array}\right) .
\end{aligned}
$$

Consider the Jordan canonical form of the matrix $X$ :

$$
X=Q\left(\begin{array}{cc}
J_{+} & 0 \\
0 & J_{-}
\end{array}\right) Q^{-1}
$$

where $J_{+}\left(J_{-}\right)$corresponds to the Jordan block associated to the eigenvalues of $X$ on the right (left) half-plane. Using the expression (13) of $X$, the decomposition (12) of $\mathcal{A}$ reduces to

$$
\mathcal{A}=\mathcal{Q} \mathcal{J} \mathcal{Q}^{-1}
$$

where

$$
\left.\mathcal{Q}=\left(\begin{array}{cc}
Q & 0 \\
0 & Q
\end{array}\right)\left[\begin{array}{cc}
\Lambda_{+} & 0 \\
0 & \Lambda_{-}
\end{array}\right)+\sqrt{\frac{p}{2}} I_{n}-\left(\begin{array}{cc}
\Lambda_{+} & 0 \\
0 & \Lambda_{-}
\end{array}\right)-\sqrt{\frac{p}{2}} I_{n}\right]
$$

and

$$
\mathcal{J}=\left[\begin{array}{cc}
\left(\begin{array}{cc}
J_{+} & 0 \\
0 & J_{-}
\end{array}\right) & 0 \\
0 & -\left(\begin{array}{cc}
J_{+} & 0 \\
0 & J_{-}
\end{array}\right)-2 \sqrt{\frac{p}{2}} I_{n}
\end{array}\right] .
$$

Now the expression of $\mathcal{P}$ is simply

$$
\mathcal{P}=\mathcal{Q}\left(\begin{array}{cc}
I_{k} & 0 \\
0 & 0
\end{array}\right) \mathcal{Q}^{-1}
$$

\footnotetext{
${ }^{1}$ the properties of the mapping $\varphi$ ensure the uniqueness of $X$ in $\mathcal{D}$
} 
where $k$ is the order of the matrix $J_{+}$. The relation (15) can easily be written in the form (8) with

$$
\begin{gathered}
\mathcal{P}_{1}=\mathcal{P}_{4}=Q\left(\begin{array}{cc}
\frac{1}{2} I_{k} & 0 \\
0 & 0
\end{array}\right) Q^{-1} \equiv \frac{1}{2} P \\
\mathcal{P}_{2}=Q\left(\begin{array}{cc}
\frac{1}{2}\left(J_{+}+\sqrt{\frac{p}{2}} I_{k}\right) & 0 \\
0 & 0
\end{array}\right) Q^{-1} \equiv \frac{1}{2}(P A)^{\frac{1}{2}}
\end{gathered}
$$

and

$$
\mathcal{P}_{3}=Q\left(\begin{array}{cc}
\frac{1}{2}\left(J_{+}+\sqrt{\frac{p}{2}} I_{k}\right)^{-1} I_{n} & 0 \\
0 & 0
\end{array}\right) Q^{-1}
$$

It remains only to observe that by the continuity arguments, that is, by considering, for example, the matrix $A_{\epsilon}=A+\epsilon I_{n}$, one can remove the assumption about nonsingularity of $A$.

\section{$3 \quad$ Elliptic dichotomy}

Let $z B-A$ be a regular matrix pencil of order $n$ having no eigenvalue on the ellipse $\Gamma$ of equation

$$
\frac{x^{2}}{a^{2}}+\frac{y^{2}}{b^{2}}=1
$$

We assume throughout this section that $a \geq b>0$. Consider the mapping $\psi: \lambda \in$ $\mathbb{C} \mapsto z \in \mathbb{C}$ defined by the formula

$$
z=\psi(\lambda)=\frac{(a+b) \lambda^{2}+(a-b)}{2 \lambda} .
$$

It is easy to see that $\psi$ maps the region

$$
R_{1}=\left\{\lambda \in \mathbb{C}|| \lambda \mid<\frac{a-b}{a+b}\right\} \cup\{\lambda \in \mathbb{C}|| \lambda \mid>1\}
$$

onto the exterior of $\Gamma$ and maps the annulus

$$
R_{2}=\left\{\lambda \in \mathbb{C}\left|\frac{a-b}{a+b}<\right| \lambda \mid<1\right\}
$$

onto the interior of $\Gamma$. Moreover, $\psi$ conformally transforms the exterior of the unit circle onto the exterior of the ellipse $\Gamma$. The mapping $\psi$ is a bijection when $r>\sqrt{\frac{a-b}{a+b}}$. 
In particular, $\psi$ conformally maps the exterior of the circle onto the exterior of the ellipse $\Gamma$.

Consider now the quadratic matrix pencil

$$
\frac{a+b}{2} B \lambda^{2}-A \lambda+\frac{a-b}{2} B
$$

Then

$$
\frac{a+b}{2} B \lambda^{2}-A \lambda+\frac{a-b}{2} B=\lambda(z B-A)
$$

where $z=\psi(\lambda)$ as defined in (20). This shows the relationship between the eigenstructure of the quadratic matrix pencil (21) and the eigenstructure of the original pencil $z B-A$.

¿From (22) and the assumption on the eigenvalues of $z B-A$, we see that the quadratic matrix pencil (21) have no eigenvalue on the unit circle and that

$$
\sup _{|\lambda|=1}\left\|\left[\frac{a+b}{2} B \lambda^{2}-A \lambda+\frac{a-b}{2} B\right]^{-1}\right\|=\sup _{z \in \Gamma}\left\|(z B-A)^{-1}\right\| .
$$

A classical way of dealing with the quadratic matrix pencil (21) is to consider a matrix pencil [3] of the form $\mu \mathcal{B}-\mathcal{A}$ where

$$
\mathcal{B}=\left(\begin{array}{cc}
\frac{a+b}{2} B & -A \\
0 & \frac{a+b}{2} B
\end{array}\right) \text { and } \mathcal{A}=\left(\begin{array}{cc}
-\frac{a-b}{2} B & 0 \\
A & -\frac{a-b}{2} B
\end{array}\right)
$$

It is easy to show that if $\left(\frac{a+b}{2} B \lambda^{2}-A \lambda+\frac{a-b}{2} B\right) x=0$, then $\left(\lambda^{2} \mathcal{B}-\mathcal{A}\right)\left(\begin{array}{c}\lambda x \\ x\end{array}\right)=$ 0 . From the assumption on the eigenvalues of $z B-A$, we see that the eigenvalues of the matrix pencil $\mu \mathcal{B}-\mathcal{A}$ cannot be on the unit circle. Thus the problem of verification of absence of the eigenvalues of the matrix pencil $z B-A$ on the ellipse $\Gamma$ is reduced to a spectral dichotomy problem with respect to the unit circle, and this latter problem is now well understood $[2,6,7,8]$ and can be applied efficiently.

In the spectrum dichotomy problem for the matrix pencil $z B-A$, the quality of the dichotomy is characterized by the numerical parameter $[7,8,1]$

$$
\alpha=\sup _{z \in \Gamma}\left\|(z B-A)^{-1}\right\| .
$$

Similarly, the quality of the dichotomy for the matrix pencil $\mu \mathcal{B}-\mathcal{A}$ with respect to the unit circle is characterized by the numerical parameter

$$
\tilde{\alpha}=\sup _{|\mu|=1}\left\|(\mu \mathcal{B}-\mathcal{A})^{-1}\right\| .
$$


As in the previous section, we start by comparing the two quantities $\alpha$ and $\tilde{\alpha}$

Proposition 3.1 The quantities $\alpha$ and $\tilde{\alpha}$ defined in (24) and (25) are equal:

$$
\alpha=\tilde{\alpha}
$$

Proof : We have

$$
\begin{aligned}
& \left(\mu^{2} \mathcal{B}-\mathcal{A}\right)^{-1}=\left(\begin{array}{cc}
z \mu B & -A \mu^{2} \\
-A & z \mu B
\end{array}\right)^{-1} \text { with } z=\psi(\mu) \\
& =\left(\begin{array}{cc}
I_{n} & 0 \\
0 & \mu^{-1} I_{n}
\end{array}\right)\left(\begin{array}{cc}
z B & -A \\
-A & z B
\end{array}\right)^{-1}\left(\begin{array}{cc}
\mu^{-1} I_{n} & 0 \\
0 & I_{n}
\end{array}\right) \\
& =\left(\begin{array}{cc}
I_{n} & 0 \\
0 & \mu^{-1} I_{n}
\end{array}\right) \\
& \times \frac{1}{2}\left[\begin{array}{cc}
(z B-A)^{-1}+(z B+A)^{-1} & (z B-A)^{-1}-(z B+A)^{-1} \\
(z B-A)^{-1}-(z B+A)^{-1} & (z B-A)^{-1}+(z B+A)^{-1}
\end{array}\right] \\
& \times\left(\begin{array}{cc}
\mu^{-1} I_{n} & 0 \\
0 & I_{n}
\end{array}\right) \\
& =\left(\begin{array}{cc}
I_{n} & 0 \\
0 & \mu^{-1} I_{n}
\end{array}\right)\left(\begin{array}{cc}
\frac{I_{n}}{\sqrt{2}} & -\frac{I_{n}}{\sqrt{2}} \\
\frac{I_{n}}{\sqrt{2}} & \frac{I_{n}}{\sqrt{2}}
\end{array}\right)\left[\begin{array}{cc}
(z B-A)^{-1} & 0 \\
0 & (z B+A)^{-1}
\end{array}\right] \\
& \times\left(\begin{array}{cc}
\frac{I_{n}}{\sqrt{2}} & \frac{I_{n}}{\sqrt{2}} \\
-\frac{I_{n}}{\sqrt{2}} & \frac{I_{n}}{\sqrt{2}}
\end{array}\right)\left(\begin{array}{cc}
\mu^{-1} I_{n} & 0 \\
0 & I_{n}
\end{array}\right)
\end{aligned}
$$

We now describe how to obtain a solution to the spectral dichotomy problem for the pencil $z B-A$ with respect to the ellipse $\Gamma$ having calculated a solution to the spectral dichotomy problem for the pencil $\mu \mathcal{B}-\mathcal{A}$ with respect to the unit circle. Let $P \in \mathbf{C}^{\mathbf{n} \times \mathbf{n}}$ be the projection matrix onto the right eigenspace of the pencil $z B-A$ associated with the eigenvalues outside the ellipse $\Gamma$ and $\mathcal{P} \in \mathbf{C}^{\mathbf{2 n} \times \mathbf{2 n}}$ the projection matrix onto the right eigenspace of $\mu \mathcal{B}-\mathcal{A}$ associated with the eigenvalues outside the unit circle. The following proposition characterizes the relation between $P$ and $\mathcal{P}$.

Proposition 3.2 Let us partition $\mathcal{P}$ in the following form

$$
\mathcal{P}=\left(\begin{array}{cc}
\mathcal{P}_{1} & \mathcal{P}_{2} \\
\mathcal{P}_{3} & \mathcal{P}_{4}
\end{array}\right) \quad \text { with } \quad \mathcal{P}_{i} \in \mathbf{C}^{\mathbf{n} \times \mathbf{n}}, \quad i=1,4
$$


Then

$$
P=\mathcal{P}_{1}+\mathcal{P}_{4}
$$

Proof: Assume that the pencil $z B-A$ has no infinite eigenvalue (this restriction is removed by the continuity arguments afterwards). Let us define a solution $X$ to the matrix equation

$$
\frac{a+b}{2} B X^{2}-A X+\frac{a-b}{2} B=0,
$$

whose eigenvalues are assumed to be outside the circle of radius $\frac{a-b}{a+b}$. Since the eigenvalues $\lambda$ of $X$ are chosen by the formula (20), that is $\lambda=\frac{z+\sqrt{z^{2}-a^{2}+b^{2}}}{a+b}$, where $z=\psi(\lambda)$ is an eigenvalue of the pencil $z B-A$, the eigenvalues of $X$ corresponding to $z$ outside the ellipse $\Gamma$ are uniquely defined ${ }^{2}$.

It is easy to see that the matrix $X_{1}=\frac{a-b}{a+b} X^{-1}$ also satisfies $\frac{a+b}{2} B X_{1}^{2}-A X_{1}+$ $\frac{a-b}{2} B=0$.

Consider the Jordan canonical form of the matrix $X$ :

$$
X=Q\left(\begin{array}{cc}
J_{\infty} & 0 \\
0 & J_{0}
\end{array}\right) Q^{-1}
$$

where $J_{\infty}\left(J_{0}\right)$ corresponds to the Jordan block associated to the eigenvalues of $X$ outside (inside) the unit circle. ¿From (30) and the identity

$$
\left[\begin{array}{cc}
\frac{a-b}{2} B & 0 \\
-A & \frac{a-b}{2} B
\end{array}\right]\left[\begin{array}{cc}
X & X_{1} \\
I_{n} & I_{n}
\end{array}\right]+\left[\begin{array}{cc}
\frac{a+b}{2} B & -A \\
0 & \frac{a+b}{2} B
\end{array}\right]\left[\begin{array}{cc}
X & X_{1} \\
I_{n} & I_{n}
\end{array}\right]\left[\begin{array}{cc}
X^{2} & 0 \\
0 & X_{1}^{2}
\end{array}\right]=0
$$

we see that

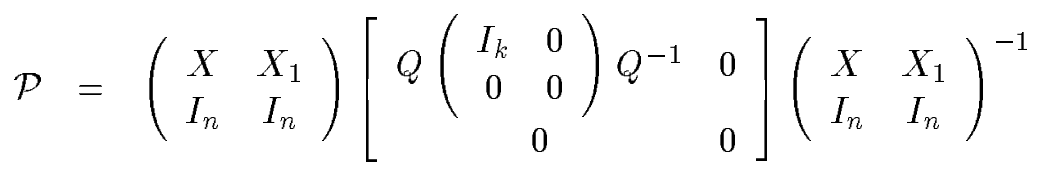

where $k$ is the order of the matrix $J_{\infty}$. Note that the matrix $X-X_{1}$ is nonsingular if and only if the matrix pencil $z B-A$ does not have $\pm \sqrt{a^{2}-b^{2}}$ as eigenvalues. This can be assumed without loss of generality (continuity arguments). Thus

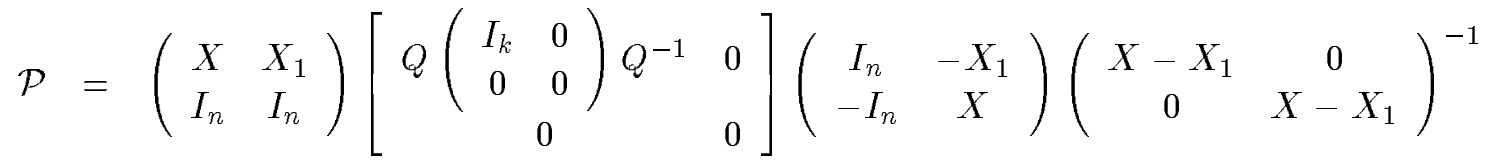

\footnotetext{
${ }^{2}$ This is due to the properties of the mapping $\psi$
} 


$$
\begin{aligned}
& =\left(\begin{array}{cc}
Q & 0 \\
0 & Q
\end{array}\right)\left[\begin{array}{cccc}
J_{\infty} & 0 & 0 & 0 \\
0 & 0 & 0 & 0 \\
I_{k} & 0 & 0 & 0 \\
0 & 0 & 0 & 0
\end{array}\right]\left[\begin{array}{cccc}
I_{k} & 0 & -\frac{a-b}{a+b} J_{\infty}^{-1} & 0 \\
0 & 0 & 0 & 0 \\
0 & 0 & 0 & 0 \\
0 & 0 & 0 & 0
\end{array}\right]\left(\begin{array}{cc}
Q & 0 \\
0 & Q
\end{array}\right)^{-1} \\
& \times\left(\begin{array}{cc}
X-X_{1} & 0 \\
0 & X-X_{1}
\end{array}\right)^{-1} \\
& =\left[\begin{array}{cc}
Q\left(\begin{array}{cc}
J_{\infty} & 0 \\
0 & 0
\end{array}\right) Q^{-1} & Q\left(\begin{array}{cc}
-\frac{a-b}{a+b} I_{k} & 0 \\
0 & 0
\end{array}\right) Q^{-1} \\
Q\left(\begin{array}{cc}
I_{k} & 0 \\
0 & 0
\end{array}\right) Q^{-1} & Q\left(\begin{array}{cc}
-\frac{a-b}{a+b} J_{\infty}^{-1} & 0 \\
0 & 0
\end{array}\right) Q^{-1}
\end{array}\right]\left(\begin{array}{cc}
X-X_{1} & 0 \\
0 & X-X_{1}
\end{array}\right)^{-1} \\
& \equiv\left(\begin{array}{ll}
\mathcal{P}_{1} & \mathcal{P}_{2} \\
\mathcal{P}_{3} & \mathcal{P}_{4}
\end{array}\right) \text {. }
\end{aligned}
$$

with

$$
\begin{aligned}
& \mathcal{P}_{1}=Q\left[\begin{array}{cc}
J_{\infty}\left(J_{\infty}-\frac{a-b}{a+b} J_{\infty}^{-1}\right)^{-1} & 0 \\
0 & 0
\end{array}\right] Q^{-1} \\
& \mathcal{P}_{2}=Q\left[\begin{array}{cc}
-\frac{a-b}{a+b}\left(J_{\infty}-\frac{a-b}{a+b} J_{\infty}^{-1}\right)^{-1} & 0 \\
0 & 0
\end{array}\right] Q^{-1} \\
& \mathcal{P}_{3}=Q\left[\begin{array}{cc}
\left(J_{\infty}-\frac{a-b}{a+b} J_{\infty}^{-1}\right)^{-1} & 0 \\
0 & 0
\end{array}\right] Q^{-1} \\
& \mathcal{P}_{4}=Q\left[\begin{array}{cc}
-\frac{a-b}{a+b} J_{\infty}^{-1}\left(J_{\infty}-\frac{a-b}{a+b} J_{\infty}^{-1}\right)^{-1} & 0 \\
0 & 0
\end{array}\right] Q^{-1}
\end{aligned}
$$

and we have $\mathcal{P}_{1}+\mathcal{P}_{4}=Q\left(\begin{array}{cc}I_{k} & 0 \\ 0 & 0\end{array}\right) Q^{-1}=P$

\section{References}

[1] Z. Bai, J. Demmel, And M. Gu, Inverse Free Parallel Spectral Divide and Conquer Algorithms for Nonsymmetric Eigenproblems, Tech. Rep., Computer Science Division Tech Report UCB/CSD-94-793, U. C. Berkeley, 1994. 
[2] A. Y. Bulgakov and S. K. Godunov, Circular Dichotomy of the Spectrum of a Matrix, Siberian Math. J., 29 (1988), pp. 734-744.

[3] F. R. Gantmacher, Théorie des matrices. Tome 2, Dunod, Paris, 1966.

[4] S. K. Godunov, Problem of the Dichotomy of the Spectrum of a Matrix, Siberian Math. J., 27 (1986), pp. 649-660.

[5] S. K. Godunov and M. Sadkane, Elliptic Dichotomy of a Matrix Spectrum, Tech. Rep., IRISA Report no 836., 1994.

[6] A. N. Malyshev, Computing Invariant Subspaces of a Regular Linear Pencil of Matrices, Siberian Math. J., 30 (1989), pp. 559-567.

[7] _ Guaranteed Accuracy in Spectral Problems of Linear Algebra,I,II, Siberian Advances in Math, 2 (1992), No. 1, pp. 144-197, No. 2, 153-204.

[8] — Parallel Algorithm for Solving Some Spectral Problems of Linear Algebra, Lin. Alg. Appl., 188,189 (1993), pp. 489-520.

[9] C. F. Van Loan, How Near is a Stable Matrix to an Unstable Matrix, Contemporary Mathematics, 47 (1985), pp. 465-477. 


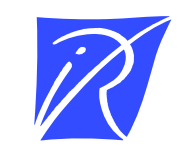

Unité de recherche INRIA Lorraine, Technopôle de Nancy-Brabois, Campus scientifique, 615 rue du Jardin Botanique, BP 101, 54600 VILLERS LÈS NANCY

Unité de recherche INRIA Rennes, Irisa, Campus universitaire de Beaulieu, 35042 RENNES Cedex

Unité de recherche INRIA Rhône-Alpes, 46 avenue Félix Viallet, 38031 GRENOBLE Cedex 1

Unité de recherche INRIA Rocquencourt, Domaine de Voluceau, Rocquencourt, BP 105, 78153 LE CHESNAY Cedex

Unité de recherche INRIA Sophia-Antipolis, 2004 route des Lucioles, BP 93, 06902 SOPHIA-ANTIPOLIS Cedex

Éditeur

INRIA, Domaine de Voluceau, Rocquencourt, BP 105, 78153 LE CHESNAY Cedex (France)

ISSN 0249-6399 